\title{
Resonances and dipole moments in dielectric, magnetic, and magnetodielectric
} cylinders

an overview

Dirksen, A.; Arslanagic, Samel; Breinbjerg, Olav

Published in:

Applied Physics A: Materials Science \& Processing

Link to article, DOI:

10.1007/s00339-010-6219-6

Publication date:

2011

Document Version

Publisher's PDF, also known as Version of record

Link back to DTU Orbit

Citation (APA):

Dirksen, A., Arslanagic, S., \& Breinbjerg, O. (2011). Resonances and dipole moments in dielectric, magnetic, and magnetodielectric cylinders: an overview. Applied Physics A: Materials Science \& Processing, 103, $789-793$. https://doi.org/10.1007/s00339-010-6219-6

\section{General rights}

Copyright and moral rights for the publications made accessible in the public portal are retained by the authors and/or other copyright owners and it is a condition of accessing publications that users recognise and abide by the legal requirements associated with these rights.

- Users may download and print one copy of any publication from the public portal for the purpose of private study or research.

- You may not further distribute the material or use it for any profit-making activity or commercial gain

- You may freely distribute the URL identifying the publication in the public portal 


\title{
Resonances and dipole moments in dielectric, magnetic, and magnetodielectric cylinders-an overview
}

\author{
A. Dirksen · S. Arslanagic • O. Breinbjerg
}

Received: 15 January 2010 / Accepted: 3 December 2010 / Published online: 4 January 2011

(c) Springer-Verlag 2011

\begin{abstract}
An eigenfunction solution to the problem of plane wave scattering by dielectric, magnetic, and magnetodielectric cylinders is used for a systematic investigation of their resonances. An overview of the resonances with electric and magnetic dipole moments, needed in, e.g., the synthesis of metamaterials, is given with an emphasis on their strength, bandwidth, and isolation.
\end{abstract}

\section{Introduction}

Among a large variety of metamaterial (MTM) realizations, see, e.g., [1] and the works referenced therein, special attention has recently been devoted to designs based on cylindrical or spherical inclusions of dielectric or magnetodielectric materials [2-4]. One main advantage of these designs, as compared to arrangements of conducting wires and split ring resonators [1], is their potential of providing low-loss and isotropic MTMs. The basics of the novel MTM realizations rest upon the excitation of electric and magnetic dipole moments in the dielectric or magnetodielectric inclusions, which give the possibility of achieving negative effective permittivity and permeability. In consequence hereof, there is an increased interest in a detailed understanding of the underlying physics of these resonances. The present work gives an overview of the resonances providing electric and magnetic dipole moments in dielectric, magnetic, and magnetodielectric cylinders illuminated by a uniform plane

A. Dirksen · S. Arslanagic (凶) $\cdot$ O. Breinbjerg Department of Electrical Engineering, Electromagnetic Systems, Technical University of Denmark, Build. 348, Ørsteds Plads, 2800 Kgs. Lyngby, Denmark

e-mail: sar@elektro.dtu.dk wave. Using the eigenfunction solution, the resonant properties are illustrated for a variety of cylinders. In particular, the strength, bandwidth, and isolation of the resonances, these being crucial parameters in MTM designs, are investigated. Moreover, analytical expressions are presented for electric and magnetic dipole moments in all cylinders, thus providing additional insight into the potential of such inclusions for MTM design. Throughout the manuscript, the time factor $\exp (j \omega t)$, with $\omega$ being the angular frequency and $t$ being the time, is assumed and suppressed.

\section{Configuration and theory}

The cross-section of the configuration is shown in Fig. 1. It consists of an infinite circular cylinder illuminated by a plane wave and immersed into free-space with the permittivity $\varepsilon_{0}$, permeability $\mu_{0}$, and the wave number $\beta_{0}=$ $\omega \sqrt{\varepsilon_{0} \mu_{0}}=2 \pi / \lambda_{0}$, with $\lambda_{0}$ being the free-space wavelength. The cylinder has a radius $a$ and is made of a simple and lossy material with the permittivity $\varepsilon_{c}=\varepsilon_{0} \varepsilon_{r}=\varepsilon_{0}\left(\varepsilon_{r}^{\prime}-j \varepsilon_{r}^{\prime \prime}\right)$, permeability $\mu_{c}=\mu_{0} \mu_{r}=\mu_{0}\left(\mu_{r}^{\prime}-j \mu_{r}^{\prime \prime}\right)$, and wave number $\beta=\omega \sqrt{\varepsilon_{c} \mu_{c}}$ with $\operatorname{Im}\{\beta\} \leq 0$. A cylindrical coordinate system $(\rho, \phi, z)$, and the associated Cartesian coordinate system $(x, y, z)$ are introduced such that the $z$-axis coincides

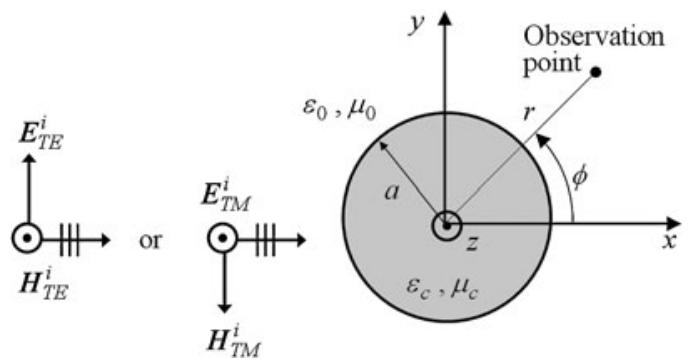

Fig. 1 The configuration 
with the axis of the cylinder, and the incident wave is propagating along the positive $x$-direction. Two polarizations of the incident wave are considered: transverse electric $\left(\mathrm{TE}^{z}\right)$ and transverse magnetic $\left(\mathrm{TM}^{z}\right)$. The exact solution to these problems is based on the eigenfunction expansion technique and is rather straightforward to obtain, see, e.g., [5]. For TE polarization, $\boldsymbol{H}_{\mathrm{TE}}^{i}$ denotes the known magnetic field incident on the cylinder, $\boldsymbol{H}_{\mathrm{TE}}^{s}$ denotes the magnetic field scattered by the cylinder, and $\boldsymbol{H}_{\mathrm{TE}}^{t}$ denotes the total magnetic field inside the cylinder; moreover, $\boldsymbol{E}_{\mathrm{TE}}^{i}, \boldsymbol{E}_{\mathrm{TE}}^{s}$, and $\boldsymbol{E}_{\mathrm{TE}}^{t}$ denote the corresponding electric fields. The unknown fields $\left(\boldsymbol{H}_{\mathrm{TE}}^{s}, \boldsymbol{E}_{\mathrm{TE}}^{s}\right)$ and $\left(\boldsymbol{H}_{\mathrm{TE}}^{t}, \boldsymbol{E}_{\mathrm{TE}}^{t}\right)$ are expanded in cylindrical wave functions with the unknown expansion coefficients $a_{n}$ and $b_{n}$, respectively, where $n$ is the mode number (i.e., $n=0$ is the monopole mode, $n=1$ is the dipole mode, etc.). For $\mathrm{TM}^{z}$ polarization, $\boldsymbol{E}_{\mathrm{TE}}^{i}$ denotes the electric field incident on the cylinder, $\boldsymbol{E}_{\mathrm{TE}}^{s}$ is the electric field scattered by the cylinder, and $\boldsymbol{E}_{\mathrm{TE}}^{t}$ is the total electric field inside the cylinder; moreover, $\boldsymbol{H}_{\mathrm{TE}}^{i}, \boldsymbol{H}_{\mathrm{TE}}^{s}$, and $\boldsymbol{H}_{\mathrm{TE}}^{t}$ denote the corresponding magnetic fields. The unknown fields $\left(\boldsymbol{E}_{\mathrm{TE}}^{s}, \boldsymbol{H}_{\mathrm{TE}}^{s}\right)$ and $\left(\boldsymbol{E}_{\mathrm{TE}}^{t}, \boldsymbol{H}_{\mathrm{TE}}^{t}\right)$ are expanded in cylindrical wave functions with the unknown expansion coefficients $d_{n}$ and $e_{n}$, respectively. All expansion coefficients are found by use of the boundary conditions at the cylinder surface. With this solution, the different electric and magnetic dipole moments can be calculated. For a dielectric cylinder $\left(\varepsilon_{r} \neq 1, \mu_{r}=1\right)$, the electric and magnetic dipole moments are given by

$\boldsymbol{p}_{d}=\frac{1}{j \omega} \int_{V} \boldsymbol{J}_{p}\left(\boldsymbol{r}^{\prime}\right) d v^{\prime}, \quad$ and

$\boldsymbol{m}_{d}=\frac{1}{2} \int_{V} \boldsymbol{r}^{\prime} \times \boldsymbol{J}_{p}\left(\boldsymbol{r}^{\prime}\right) d v^{\prime}$,

respectively, where $\boldsymbol{J}_{p}=j \omega\left(\varepsilon_{c}-\varepsilon_{0}\right) \boldsymbol{E}_{\mathrm{TE}, \mathrm{TM}}^{t}$ is the polarization current density inside the cylinder, and $d v^{\prime}$ denotes a differential volume element with the position vector $\boldsymbol{r}^{\prime}$. For a magnetic cylinder $\left(\varepsilon_{r}=1, \mu_{r} \neq 1\right)$, the electric and magnetic dipole moments are given by

$\boldsymbol{p}_{m}=-\frac{\varepsilon_{0}}{2} \int_{V} \boldsymbol{r}^{\prime} \times \boldsymbol{J}_{m}\left(\boldsymbol{r}^{\prime}\right) d v^{\prime}, \quad$ and

$\boldsymbol{m}_{m}=\frac{1}{j \omega \mu_{0}} \int_{V} \boldsymbol{J}_{m}\left(\boldsymbol{r}^{\prime}\right) d v^{\prime}$,

where $\boldsymbol{J}_{m}=j \omega\left(\mu_{c}-\mu_{0}\right) \boldsymbol{H}_{\mathrm{TE}, \mathrm{TM}}^{t}$ is the magnetization current density inside the cylinder. In a magnetodielectric cylin$\operatorname{der}\left(\varepsilon_{r} \neq 1, \mu_{r} \neq 1\right)$, both electric and magnetic polarization currents exist, and the total electric and magnetic dipole moments are $\boldsymbol{p}_{m d}=\boldsymbol{p}_{d}+\boldsymbol{p}_{m}$ and $\boldsymbol{m}_{m d}=\boldsymbol{m}_{d}+\boldsymbol{m}_{m}$, respectively. An electric dipole moment can be created by an electric line current or a magnetic loop current while a magnetic dipole moment can be created by an electric loop current or a magnetic line current. These line and loop currents are associated with the $n=0$ and $n=1$ modes which are thus of relevance in the design of MTMs.

\section{Numerical results}

The expansion coefficients $a_{n}$ and $b_{n}$, as well as $d_{n}$ and $e_{n}$, depend on $\beta_{0} a, \varepsilon_{r}$ and $\mu_{r}$. For specific values of these parameters, the coefficients attain a large value which implies a resonance of the corresponding mode. The resonant properties of a lossless dielectric cylinder with $\mathrm{TE}^{z}$ polarization are illustrated in Fig. 2 for the $n=0$ and $n=1$ modes. Figures 2(a)-(d) show the magnitudes of $a_{0}, b_{0}, a_{1}$, and $b_{1}$, respectively, as a function of $\sqrt{\varepsilon_{r}}$ and $\beta_{0} a$ (the default color axis has been changed in (b) and (d) to enhance the visibility of the results). It is seen that the maxima of the coefficients follow specific resonance curves; the resonances are designated as the $\mathrm{TE}_{n l}^{d}$ resonances, where for each $n, l=0$ corresponds to the leftmost curve, $l=1$ corresponds to the second leftmost curve, etc. Thus, Figs. 2(a)-(b) show the $\mathrm{TE}_{0 l}^{d}$ resonances, and Figs. 2(c)-(d) show the $\mathrm{TE}_{1 l}^{d}$ resonances. The designations $\operatorname{TE}_{n l}^{d}\left(a_{n}\right)$ and $\operatorname{TE}_{n l}^{d}\left(b_{n}\right)$ are used to refer to a specific coefficient since internal and external resonances do not necessarily occur for the same values of $\beta_{0} a, \varepsilon_{r}$ and $\mu_{r}$. Following a resonance curve, e.g., the $\mathrm{TE}_{01}^{d}$ resonance, it is seen how a higher $\varepsilon_{r}$ results in a smaller $\beta_{0} a$ (implying a smaller radius $a$ at a given frequency) and a noticeable narrowing of the resonances. Moreover, for a given $\varepsilon_{r}$, the $\mathrm{TE}_{1 l}^{d}$ resonances occur at a larger value of $\beta_{0} a$ than the $\mathrm{TE}_{0 l}^{d}$ resonances.

Figures 2(e)-(h) show the magnitudes of $a_{0}, b_{0}, a_{1}$, and $b_{1}$, respectively, as a function of $\beta_{0} a$ for specific values of $\sqrt{\varepsilon_{r}}$. This corresponds to horizontal cuts in Figs. 2(a)-(d) but with a reduced range of $\beta_{0} a$. The width of the resonances for the $n=0$ mode is seen to be broader than for the $n=1$ mode, and for each of the modes the width differs for different resonances for a given $\sqrt{\varepsilon_{r}}$. In addition, the higher the value of $\sqrt{\varepsilon_{r}}$ the narrower is the width, and the higher is the amplitude of the internal $b_{0}$ and $b_{1}$ coefficients. It is interesting that all the resonances of the external coefficients $a_{0}$ and $a_{1}$ have the same amplitude; however, this will be different for other types of illumination and normalization of expansion functions. Although not shown inhere, it was found that the inclusion of moderate losses lowers the amplitude of the resonances, shifts slightly their position in frequency, and broadens their width.

With the aim of exploiting the resonances for MTM realizations, it is important that the $n=0$ and $n=1$ modes are sufficiently dominant, i.e., their expansion coefficients at resonance should be large compared to those of other modes. Figure 2(i) shows the isolation of the $n=0$ mode in terms of the quantity $\left|a_{1} / a_{0}\right|$ for the $\mathrm{TE}_{01}^{d}\left(a_{0}\right)$ resonance, and $\left|b_{1} / b_{0}\right|$ for the $\mathrm{TE}_{01}^{d}\left(b_{0}\right)$ resonance as a function of $\sqrt{\varepsilon_{r}^{\prime}}$, while the isolation of the $n=1$ mode is shown in Fig. 2(j) in terms of $\left|a_{0} / a_{1}\right|$ and $\left|a_{2} / a_{1}\right|$, evaluated at the $\operatorname{TE}_{11}^{d}\left(a_{1}\right)$ resonance, and $\left|b_{0} / b_{1}\right|$ and $\left|b_{2} / b_{1}\right|$ for the $\mathrm{TE}_{11}^{d}\left(b_{1}\right)$ resonance. In Figs. 2(i)-(j), a curve with a given color appears 

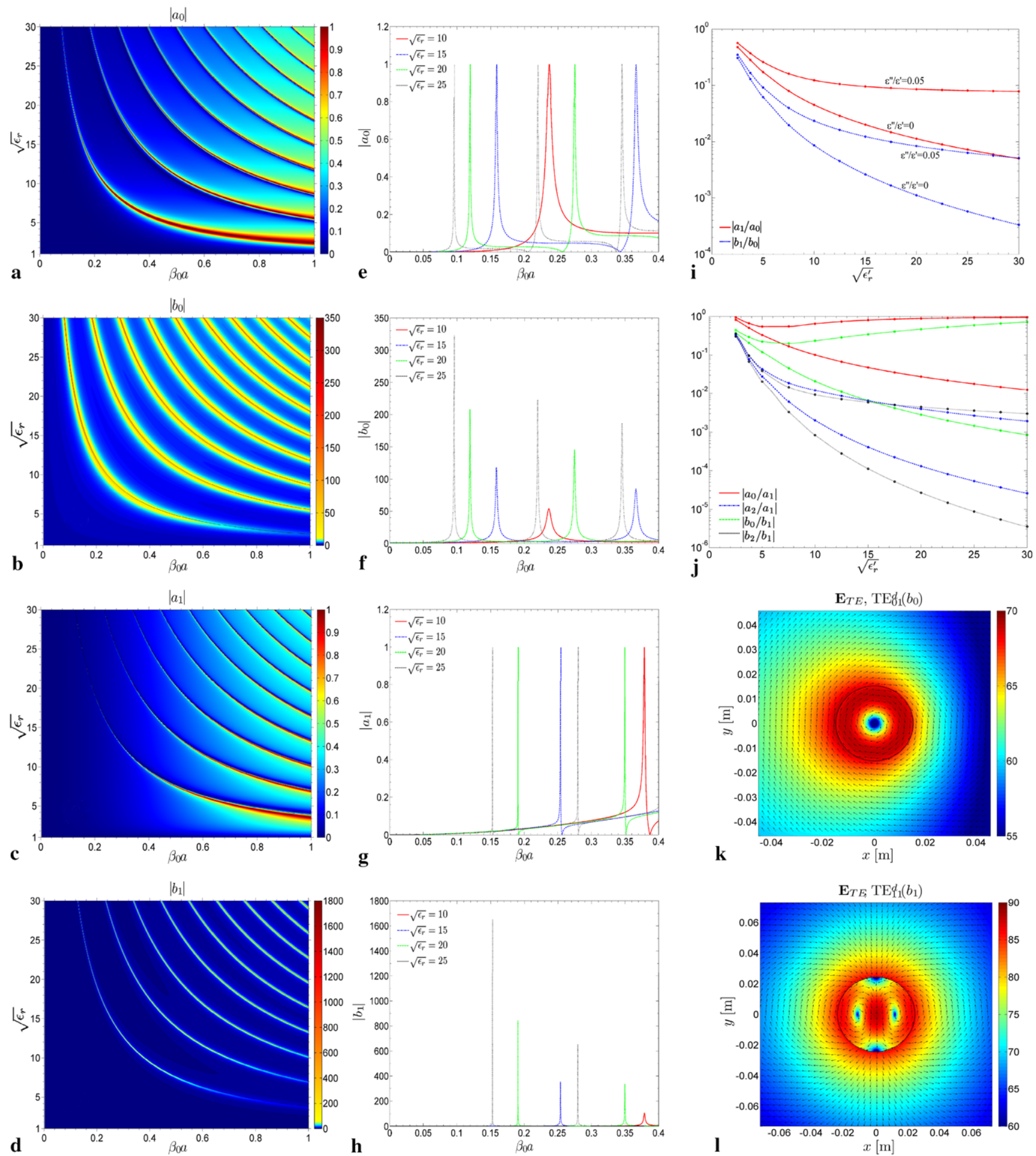

Fig. 2 Resonant properties of a dielectric cylinder with $\mathrm{TE}^{z}$ polarization. The magnitude of the expansion coefficients as a function of $\sqrt{\varepsilon_{r}}$ and $\beta_{0} a$ (a)-(d), and as a function of $\beta_{0} a$ for specific values of $\sqrt{\varepsilon_{r}}$ (e)-(h). The isolation properties of the $n=0$ and $n=1$ modes $(\mathbf{i})-(\mathbf{j})$, and the magnitude and the direction of the total electric fields at the $\mathrm{TE}_{01}^{d}\left(b_{0}\right)$ and $\mathrm{TE}_{11}^{d}\left(b_{1}\right)$ resonances inside and outside the cylinders for the $n=0$ and $n=1$ modes $(\mathbf{k})-(\mathbf{l})$. By duality, the shown results also apply for a magnetic cylinder with $\mathrm{TM}^{z}$ polarization. The corresponding quantities for the dual case are obtained by performing the substitutions: $a_{0} \rightarrow d_{0}, a_{1} \rightarrow d_{1}, b_{0} \rightarrow e_{0}, b_{1} \rightarrow e_{1}, \varepsilon_{r} \rightarrow \mu_{r}, \boldsymbol{E}_{\mathrm{TE}} \rightarrow$ $-\sqrt{\mu_{0} / \varepsilon_{0}} \boldsymbol{H}_{\mathrm{TM}}, \mathrm{TE}_{01}^{d}\left(b_{0}\right) \rightarrow \mathrm{TM}_{01}^{m}\left(e_{0}\right)$, and $\mathrm{TE}_{11}^{d}\left(b_{1}\right) \rightarrow \mathrm{TM}_{11}^{m}\left(e_{1}\right)$ 
twice; the lower (upper) curve corresponds to the lossless (lossy) case for which $\varepsilon_{r}^{\prime \prime} / \varepsilon_{r}^{\prime}=0\left(\varepsilon_{r}^{\prime \prime} / \varepsilon_{r}^{\prime}=0.05\right)$. In the lossless cases, larger $\varepsilon_{r}^{\prime}$ results in a more isolated mode, whereas the isolation decreases as the loss is included. As an example, it is seen in Fig. 2(i) that for $\sqrt{\varepsilon_{r}^{\prime}}=5$ and zero loss, the exterior (interior) coefficient $a_{1}\left(b_{1}\right)$ is about $18 \%(6 \%)$ of $a_{0}\left(b_{0}\right)$, whereas for $\sqrt{\varepsilon_{r}^{\prime}}=25$ it is only about $0.8 \%(0.06 \%)$ of $a_{0}\left(b_{0}\right)$. It is interesting that the isolation of the $n=1$ mode in the lossy case is, in fact, worsened as $\varepsilon_{r}^{\prime}$ increases. In general, the isolation properties for a given $\varepsilon_{r}^{\prime}$ are better for the $n=0$ mode than for the $n=1$ mode; the former mode also has a wider width of the resonances. Moreover, a trade-off between the width and isolation of the resonances must be made since an increase of $\varepsilon_{r}^{\prime}$ decreases the width while increasing the isolation for lossless cylinders.

The above results are next confirmed with field illustrations. Figure $2(\mathrm{k})$ shows, for a resonance frequency of $f_{0}=300 \mathrm{MHz}$, the magnitude (color) and the direction at the time $t=0$ (arrows) of the total electric field for a dielectric cylinder $\left(\varepsilon_{r}=625\right)$ at the $\mathrm{TE}_{01}^{d}\left(b_{0}\right)$ resonance (the cylinder radius is $a=15.25 \mathrm{~mm}$ ) (specifically, the quantity $20 \log _{10}\left|\boldsymbol{E}_{\mathrm{TE}}\right|$, where $\left|\boldsymbol{E}_{\mathrm{TE}}\right|$ has been normalized with $1 \mathrm{~V} / \mathrm{m}$ is shown). The circulating electric field creates a polarization current loop which yields a magnetic dipole moment per unit length $\boldsymbol{m}_{\mathrm{TE} d}=\hat{z} H_{0} b_{0} \pi a^{2}\left(1-1 / \varepsilon_{r}\right) J_{2}(\beta a)$, with $H_{0}$ being the amplitude of the incident magnetic field and $J_{2}(\cdot)$ being the Bessel function of the second order. For the $n=1$ mode, the magnitude and the direction (for $t=0)$ of the total electric field at the $\mathrm{TE}_{11}^{d}\left(b_{1}\right)$ resonance are shown in Fig. 2(1) (the cylinder radius is $a=24.35 \mathrm{~mm}$ ). This field leads to an electric dipole moment per unit length $\boldsymbol{p}_{\mathrm{TE} d}=\hat{y} H_{0} b_{1} j 2 \pi a\left(1-1 / \varepsilon_{r}\right) J_{1}(\beta a) / \omega$, where $J_{1}(\cdot)$ is the Bessel function of the first order. We note from Fig. 2(k) that the field magnitude outside the cylinder is asymmetric with respect to $x=0$, whereas this is not the case for the corresponding field in Fig. 2(1). This difference is due to the fact that the scattered field for the $n=0$ mode is oppositely directed at any two diametrically opposite points located in the $x y$-plane, whereas it is similarly directed for the scattered field of the $n=1$ mode. Since $f_{0}=300 \mathrm{MHz}$, the incident electric field has only a small phase change across the region nearby the cylinder. Consequently, it engages in an interference of a different type with the scattered field on the two sides of the $y z$-plane for the $n=0$ mode, whereas the interference will be of the same type for the $n=1$ mode. It is important to note that the above results also hold for a magnetic cylinder with $\mathrm{TM}^{z}$ polarization due to duality. The exact correspondence between the various quantities in the two cases is found in the caption of Fig. 2. Although not shown inhere, the above results are qualitatively similar though quantitatively different for dielectric cylinders for $\mathrm{TM}^{z}$ polarization and magnetodielectric cylinders for either of the two polarizations. In particular, the respective resonances for $\mathrm{TM}^{z}$
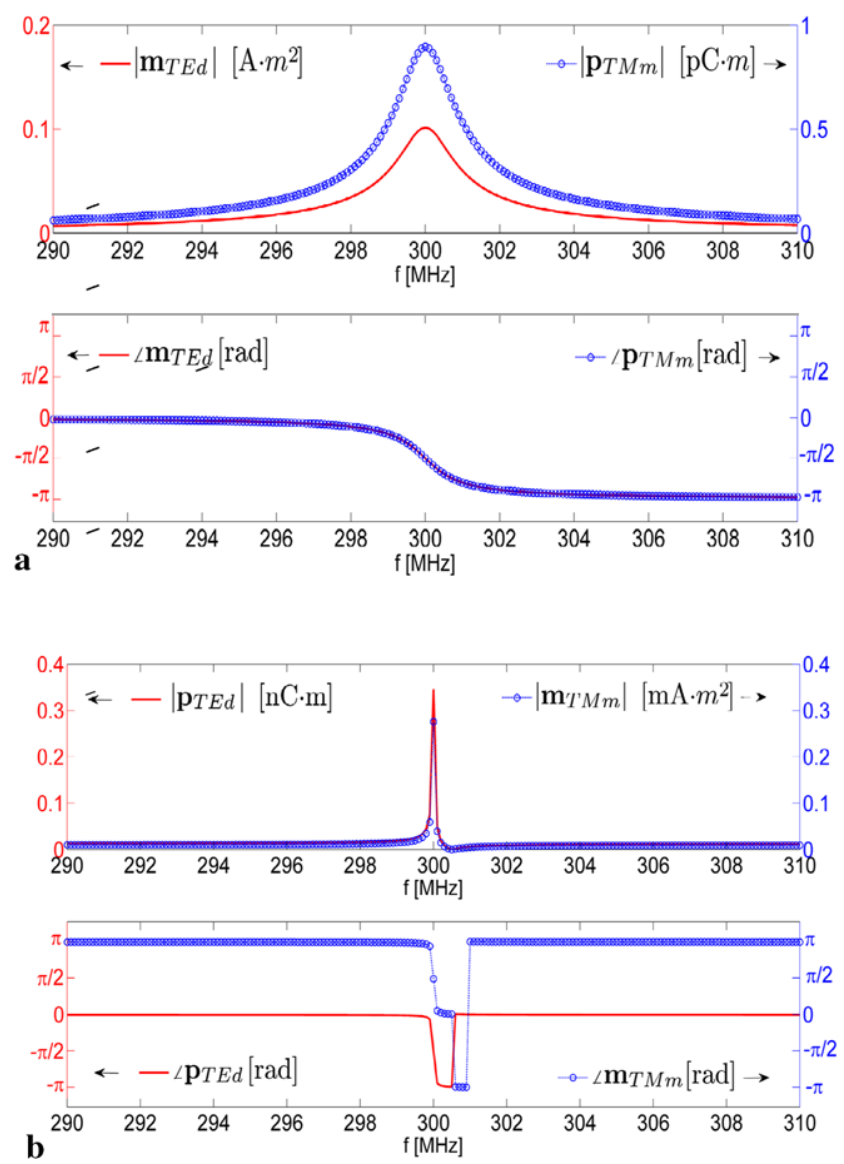

Fig. 3 Magnitude and phase of (a) $\boldsymbol{m}_{\mathrm{TE} d}$ (left vertical axis) and $\boldsymbol{p}_{\mathrm{TM} m}$ (right vertical axis), and of (b) $\boldsymbol{p}_{\mathrm{TE} d}$ (left vertical axis) and $\boldsymbol{m}_{\mathrm{TM} m}$ (right vertical axis)

polarization in these cylinders are in general broader than those shown in Figs. 2(a)-(h) and their isolation properties are somewhat poorer than those in Figs. 2(i)-(j).

Due to duality, Figs. 2(k) and 2(l) therefore also show the quantity $-\sqrt{\mu_{0} / \varepsilon_{0}} \boldsymbol{H}_{\mathrm{TM}}$ for a lossless magnetic cylin$\operatorname{der}\left(\mu_{r}=625\right)$ with $\mathrm{TM}^{z}$ polarization for the modes $n=0$ and $n=1$, respectively. The former leads to an electric dipole moment per unit length $\boldsymbol{p}_{\mathrm{TM} m}$, and the latter one to a magnetic dipole moment per unit length $\boldsymbol{m}_{\mathrm{TM} m}$. These are given by $\boldsymbol{p}_{\mathrm{TM} m}=\hat{z} E_{0} e_{0} \pi a^{2} \varepsilon_{0}\left(1-1 / \mu_{r}\right) J_{2}(\beta a)$, where $E_{0}$ is the amplitude of the incident electric field, and $\boldsymbol{m}_{\mathrm{TM} m}=$ $-\hat{y} E_{0} e_{1} j 2 \pi a\left(1-1 / \mu_{r}\right) J_{1}(\beta a) / \omega \mu_{0}$. For the cylinders treated in Figs. 2(k) and 2(l), the magnitude and phase of $\boldsymbol{m}_{\mathrm{TE} d}\left[\boldsymbol{p}_{\mathrm{TM} m}\right]$ are shown in Fig. 3(a) (vertical left [right] axis), while the corresponding results for $\boldsymbol{p}_{\mathrm{TE} d}\left[\boldsymbol{m}_{\mathrm{TM} m}\right]$ are shown in Fig. 3(b) (vertical left [right] axis). In all cases, the magnitude of the dipole moments peaks at the resonance frequency across which a phase shift of $\pi$ also occurs (the additional phase shifts in Fig. 3(b) at a frequency slightly larger than the resonance frequency are due to zero magnitudes of the two dipole moments at this frequency). The latter implies a change in the direction of the dipole mo- 
ments at the resonance frequency, whereby these become oppositely directed to the incident field, thus rendering such cylinders applicable as inclusions in a MTM design.

\section{Summary and conclusions}

An overview of electric and magnetic dipole resonances was given for dielectric and magnetic cylinders with an emphasis on their strength, bandwidth, and isolation. In general, a higher material parameter was found to lead to larger amplitude of the resonances, while narrowing the bandwidth, but improving their isolation which is better for the monopole than for the dipole mode. Although the numerical results illustrated the resonant properties of specific cylinders and specific polarizations, the approach of this manuscript provides a means of devising dielectric, magnetic, and/or magnetodielectric cylinders capable of exciting the dipole moments, of which a complete characterization, in terms of the strength, bandwidth, and isolation of the resonances, can be made for both polarizations.
Acknowledgements This work is supported by the Danish Research Council for Technology and Production Sciences within the TopAnt project.

\section{References}

1. N. Engheta, R.W. Ziolkowski, Metamaterials: Physics and Engineering Applications (Wiley, New York, 2006)

2. C. Holloway, E. Kuester, J. Baker-Jarvis, P. Kabos, A double negative (dng) composite medium composed of magnetodielectric spherical particle embedded in a matrix. IEEE Trans. Antennas Propag. 51, 2596-2603 (2003)

3. I. Vendik, O. Vendik, M. Odit, Isotropic artificial media with simultaneously negative permittivity and permeabiliy. Microw. Opt. Technol. Lett. 48, 2553-2556 (2006)

4. L. Peng, L. Ran, H. Chen, H. Zhang, J.A. Kong, T.M. Grzegorczyk, Experimental observation of left-handed behavior in an array of standard dielectric resonators. Phys. Rev. Lett. 98, 157403 (2007)

5. C.A. Balanis, Advanced Engineering Electromagnetics (Wiley, New York, 1989) 